\title{
Cardiac catheterization in pediatric pulmonary hypertension: a systematic and practical approach
}

\author{
Michael Kaestner ${ }^{1}$, Christian Apitz ${ }^{1}$, Astrid Elisabeth Lammers ${ }^{2}$ \\ ${ }^{1}$ University Children's Hospital Ulm, Division of Pediatric Cardiology, Ulm, Germany; ${ }^{2}$ University Children's Hospital Münster, Division of \\ Pediatric Cardiology, Albert-Schweitzer-Campus 1, Münster, Germany \\ Contributions: (I) Conception and design: M Kaestner; (II) Administrative support: None; (III) Provision of study materials or patients: None; (IV) \\ Collection and assembly of data: M Kaestner; (V) Data analysis and interpretation: M Kaestner, C Apitz; (VI) Manuscript writing: All authors; (VII) \\ Final approval of manuscript: All authors. \\ Correspondence to: Dr. Michael Kaestner. Division of Pediatric Cardiology, University Children's Hospital Ulm, Eythstraße 24, 89075 Ulm, Germany. \\ Email: michael.kaestner@uniklinik-ulm.de.
}

\begin{abstract}
Significant progress in the understanding of the etiology, epidemiology, pathobiology and prognosis of pulmonary hypertension $(\mathrm{PH})$ has been made over the last years. Especially in the pediatric patient population the etiology of $\mathrm{PH}$ is very heterogeneous. Nevertheless, the most recent change of the definition of $\mathrm{PH}$ to a mean pulmonary artery pressure $(\mathrm{mPAP})>20 \mathrm{mmHg}$ has been accepted by pediatricians for uniformity and concordance with adult physicians. Based on the diverse underlying medical conditions leading to $\mathrm{PH}$, a comprehensive and systematic approach for diagnosis and treatment is mandatory. Cardiac catheterization remains the gold standard for invasive assessment and acute vasoreactivity testing (AVT) additionally providing detailed information about nature of $\mathrm{PH}$. In most patients repeat cardiac catheterization may be helpful for evaluation of response to targeted $\mathrm{PH}$ treatment, risk stratification and indication for lung transplantation. However, the information and results taken from cardiac catheterization should be interpreted by experienced investigators only who are familiar with confounding factors that may influence the results. Here we provide an overview of current recommendations for invasive hemodynamic evaluation in pediatric PH. We point out different patient scenarios and provide a structured approach for AVT and response interpretation.
\end{abstract}

Keywords: Cardiac catheterization; children; pulmonary hypertension (PH); vasoreactivity

Submitted Mar 28, 2020. Accepted for publication Jun 29, 2020.

doi: $10.21037 / \mathrm{cdt}-20-395$

View this article at: http://dx.doi.org/10.21037/cdt-20-395

\section{Introduction}

Pediatric pulmonary hypertension (PH) may be caused by a broad spectrum of diseases. In infants and children, one may find characteristics and symptoms similar to adult patients. Nevertheless, pediatric $\mathrm{PH}$ also has special features that are unique to age related conditions. Even in pediatric patients, pulmonary hypertensive vascular disease (PHVD) is associated with high morbidity and mortality $(1,2)$.

Once the suspicion for $\mathrm{PH}$ is made, the patient will undergo structured diagnostic workup. Details and recommendations have been published elsewhere $(2,3)$. The majority of the examinations are non-invasive. However, for the accurate measurement of hemodynamic conditions, cardiac catheterization with calculation of pulmonary vascular resistance and acute vasoreactivity testing (AVT) is the method of choice and remains the gold standard. In addition, this procedure provides supplemental information about the underlying disease of $\mathrm{PH}$ and allows risk stratification.

In general, cardiac catheterization is recommended before starting any pulmonary vasodilating therapy. However, there are many open questions regarding cardiac catheterization in pediatric $\mathrm{PH}$ : In contrast to adult $\mathrm{PH}$, 
where a standardized protocol has been established (4), there is no general agreement in pediatric patients about a standardized manner for invasive workup. Differences include -amongst others- anesthetic strategy, inhalative substances used for AVT, and interpretation of response to AVT. All of these topics have major impact on the interpretation of results from cardiac catheterization (5).

In this review article we provide an overview of current recommendations for invasive hemodynamic evaluation in pediatric $\mathrm{PH}$ and discuss the above mentioned concerns. We propose a detailed structured approach for catheterization and $\mathrm{AVT}$ and discuss response interpretation.

\section{Protocol for cardiac catheterization and AVT}

There are differences in cardiac catheterization protocols dependent on the patients' underlying diagnosis. For patients with congenital heart disease (PAH-CHD) and intracardiac shunt a different protocol is recommended than for idiopathic PH (IPAH) patients with a structurally normal heart (6).

Patients with pulmonary vein stenosis have to be excluded from this patient population $(7,8)$. This condition is often associated with the diagnosis of congenital diaphragmatic hernia and premature infants with bronchopulmonary dysplasia.

In contrast to adults, for the initial assessment of $\mathrm{PH}$ usually left and right heart catheterization is performed in both groups. Results have to be interpreted in the context of patients age, the underlying disease and clinical status.

Access through the femoral vessels (venous and arterial) is used in most centers. A transatrial puncture or transatrial access to the systemic circulation via an ASD or foramen ovale can be used instead of an arterial puncture. For particular patients, atrial septostomy may be indicated. If right atrial pressure permits, atrial septostomy should be performed for acute pressure relief in the pulmonary circulation prior to complete a full hemodynamic evaluation. Most centers perform cardiac catheterization under resting conditions with the patient awake or mildly sedated and spontaneously breathing, others use general anesthesia. Either way, maintenance of normal blood gases (pH 7.35-7.45, $\left.\mathrm{paCO}_{2} 35-45\right)$ is crucial (6). Particularly in patients with trisomy 21 or others prone to $\mathrm{CO}_{2}$ retention or sleep apnoea, intubation may be favoured to maintain stable $\mathrm{CO}_{2}$ values to achieve normoventilation and a constant stable state.

Prior to obtaining hemodynamic data it is important to carefully set the level of the pressure transducer at the level of the left atrium for zero levelling (4,9). Differences are existing regarding the recommended level of the pressure transducer. It is widely accepted to define the level of the left atrium in a patient in supine position with the pressure transducer set halfway between anterior sternum and table surface (mid thoracic line) $(4,9,10)$.

The pressure measurements are taken directly, oxygen saturations are measured using spectrophotometric analyzers. Other hemodynamic variables are calculated from the measured parameters, such as cardiac index, transpulmonary gradient (TPG) and PVR.

Pressure measurements should at least be taken from a systemic vein [superior caval vein (SVC)], pulmonary arteries and a systemic artery. For a complete and comprehensive work-up and the differentiation of pre- and postcapillary $\mathrm{PH}$, left atrial pressure and/or pulmonary capillary wedge position and left ventricular pressures should be measured. Simultaneous pressure recordings in pulmonary wedge position and left ventricle might be helpful to carefully assess the TPG.

Pulmonary arterial wedge pressure (PAWP) is recorded with an end-open catheter, often with a balloon at the catheter tip. In order to avoid injury to the pulmonary vessel wall or even rupture of a pulmonary artery the balloon should be inflated in the right atrium. Without deflating the balloon, the catheter should be pushed through the tricuspid and pulmonary valve up to the pulmonary arteries until it reaches a wedge position. Following the protocol for right heart catheterization in adult patients with $\mathrm{PH}$, the mean of three recordings should be taken as PAWP $(4,11)$. Interpretation of the pressure data has to take the high variety of cardiac and pulmonary diseases subsequently leading to PH into account (see Table 1).

In patients with congenital cardiac defects, in particular with shunt defects, oxygen saturations should be taken from the same positions where pressures are recorded to calculate pulmonary and systemic flow volumes using the Fick formula (12). In children, results are indexed to body surface area. In certain patients it might be required to measure differentiated arterial oxygen saturation in the upper and lower part of the body, e.g., patients with large PDA and right-to-left shunt (Eisenmenger circulation) or after a modified Potts' shunt.

It is important to choose a site for the arterial blood sample, that is far enough away from the anatomical level of the shunt. For example, in patients with an atrial septal defect, the sampling site for mixed venous saturation should 
Table 1 Definitions for pulmonary hypertension following the classification of PH according to the WSPH (Nice 2018) and the classification of pediatric pulmonary hypertensive vascular disease

\begin{tabular}{|c|c|}
\hline Disease & Definition \\
\hline precapillary PH & $\begin{array}{l}\text { mPAP }>20 \mathrm{mmHg} \text {, PAWP or LVEDp } \leq 15 \mathrm{mmHg}, \mathrm{PVR} \text { index } \geq 3 \mathrm{WU} \cdot \mathrm{m}^{2} \text {, diastolic TPG (DPG) } \\
\geq 7 \mathrm{mmHg} \text { (adjunct criterion) }\end{array}$ \\
\hline postcapillary PH (LV dysfunction; HFpEF) & $\begin{array}{l}\text { mPAP }>20 \mathrm{mmHg} \text {, PAWP or LVEDd } \geq 15 \mathrm{mmHg}, \text { PVR index }<3 \mathrm{WU} \cdot \mathrm{m}^{2} \text {, diastolic TPG (DPG) } \\
<7 \mathrm{mmHg}\end{array}$ \\
\hline Pulmonary arterial hypertension (PAH) & mPAP $>20 \mathrm{mmHg}$, PAWP or LVEDp $\leq 15 \mathrm{mmHg}$, PVR index $\geq 3 \mathrm{WU} \cdot \mathrm{m}^{2}$, criteria for $\mathrm{PH}$ group 1 \\
\hline Idiopathic PAH (IPAH) & $\mathrm{PAH}$ without underlying disease known to be associated with $\mathrm{PH}$ \\
\hline Heritable PAH (HPAH) & $\begin{array}{l}\text { Patient with positive genetic testing of the index patient or with positive family history for genetic } \\
\text { testing but without detected underlying disease }\end{array}$ \\
\hline $\begin{array}{l}\text { Pulmonary Hypertensive Vascular } \\
\text { Disease (PHVD) }\end{array}$ & $\begin{array}{l}\text { Patients with biventricular circulation: } \mathrm{mPAP}>20 \mathrm{mmHg} \text { and PVR index } \geq 3 \mathrm{WU} \cdot \mathrm{m}^{2} \text {. Patients with } \\
\text { univentricular circulation (e.g., cavopulmonary connection or Fontan-physiology): mean } \\
\text { TPG }>6 \mathrm{mmHg} \text { or PVR index }>3 \mathrm{WU} \cdot \mathrm{m}^{2}\end{array}$ \\
\hline
\end{tabular}

$\mathrm{PH}$, pulmonary hypertension; mPAP, mean pulmonary artery pressure; PAWP, pulmonary capillary wedge pressure; PVR, pulmonary vascular resistance; LVEDp, left ventricular end-diastolic pressure; TPG, transpulmonary pressure gradient; DPG, diastolic pressure gradient.
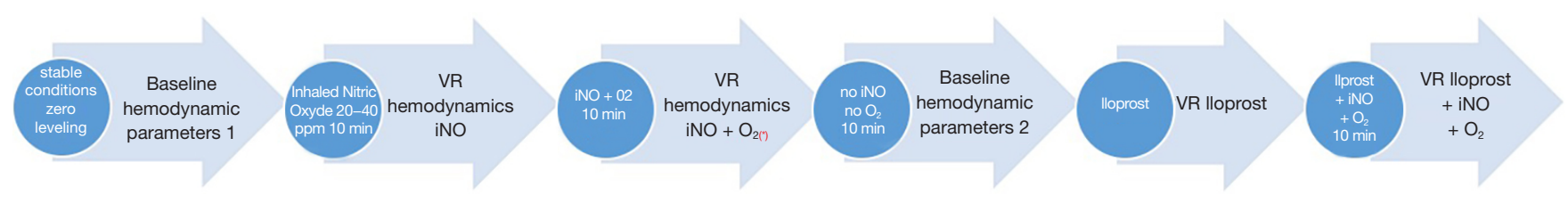

Figure 1 Suggestion of a protocol for right heart catheterization and acute vasoreactivity testing. ${ }^{*}=$ in instable patients the protocol may be stopped at this point without proceeding to the next steps for testing. VR, vasoreactivity; iNO, inhaled nitric oxide; $\mathrm{O}_{2}$, oxygen; $\mathrm{RHC}$, right heart catheterization.

be taken from the SVC. It has to be reassured that the catheter position is high enough, to avoid contamination with arterial blood due to mixing at atrial level (13).

In addition, obtaining oxygen saturations help to calculate oxygen extraction while measuring oxygen consumption is time consuming and requires special equipment and is often not used in many paediatric catheterization laboratories. Values are usually taken from tables or empiric data (14-17).

\section{Vasoreactivity testing}

A suggestion for a complete and structured workup for vasoreactivity testing is given in Figure 1. However, not all patients might be stable enough to go thru the whole testing procedure as suggested.

Oxygen, inhaled nitric oxide and nebulized prostanoids (iloprost, treprostinil) are the usually recommended substances for AVT. They seem to meet best the criteria for an "ideal" substance for AVT that is (I) short acting and has a short biological half-life and (II) targeted to pulmonary vasculature with no or very little effect on the systemic circulation and systemic ventricular function. Inhaled nitric oxide (iNO) is administered at a dose of $20-40$ ppm over 10 minutes and the effect on mean and diastolic PAP and SAP is measured and the ratio of PVR/SVR is calculated. In patients with IPAH, additional oxygen is administered 
Table 2 Medication for AVT

\begin{tabular}{lc}
\hline Drug & Dose \\
\hline iNO (inhalative) & $20-40 \mathrm{ppm}$ \\
Iloprost (inhalative) & $0.3-0.5 \mathrm{mcg} / \mathrm{kg}$ \\
Treprostinil (inhalative) & $18-36 \mathrm{mcg}$ \\
lloprost (i.v.) & $0.5-2 \mathrm{ng} / \mathrm{kg} / \mathrm{min}$ \\
Epoprostenol (i.v.) & $2-10 \mathrm{ng} / \mathrm{kg} / \mathrm{min}$, increments $2 \mathrm{ng} / \mathrm{kg} / \mathrm{min}$
\end{tabular}

iNO, inhaled nitric oxide; i.v., intravenously; ppm, parts per million; mcg, microgram; ng, nanogram.

to the inspired air $\left(\mathrm{FiO}_{2}\right.$ about 0.8$)$ and the effects are documented (6). The recommended dosage for alternative agents is given in Table 2 .

The effect of oxygen in APAH-CHD patients with an existing shunt may only result in an increase of shunt volume related to a decrease of PVR but without an actual effect on the mean pulmonary artery pressure (mPAP).

Thus, inspired oxygen should be avoided in patients with APAH-CHD as with an increase of inspired oxygen the amount of dissolved oxygen in the plasma will rise causing an additive effect to the hemoglobin bound oxygen. On oximetry only the oxygen bound to hemoglobin is measured. Subsequently, the administration of high amounts of oxygen will overestimate pulmonary blood flow and the PVR will be underestimated. If oxygen is necessary for clinical reasons with $\mathrm{FiO}_{2}$ over $0.5, \mathrm{pO}_{2}$ in the pulmonary venous blood and in the pulmonary arterial blood should be measured. The amount of dissolved oxygen in the blood should be taken into account into the calculations.

As long as the patient is tolerating the procedure in a hemodynamically stable condition, one may proceed with further hemodynamic testing. Under close monitoring of possible adverse effects of iNO and oxygen withdrawal for a period of adaption of approximately 10 minutes, new baseline hemodynamics should be documented. Then aerosolized iloprost $(0.3-0.5 \mathrm{mcg} / \mathrm{kg})$ may be given to the patient using a specific applicator and hemodynamic parameters can be obtained again. Finally, with the patient still in a stable condition iNO and $\mathrm{O}_{2}$ may be restarted to measure the cumulative effects of cyclic AMP, cyclic GMP and oxygen.

In contrast, in hemodynamically instable and critically ill patients one may aim to keep the procedure as short as possible. For those cases it might be safer to omit this last condition and reduce the testing to iNO and oxygen only, as one may treat these patients with prostacyclin irrespective of the hemodynamic reaction of this testing anyhow [see Figure $1\left(^{*}\right)$ ]. Thus, it may expose the patient to a longer procedure time with potential uncalculated risks, inability to maintain normoventilation and normocapnia without adding any additional information to the hemodynamic data, that would have consequences for the treatment decision. The risk for those adverse effects may even be higher in patients with Down syndrome.

\section{Assessment of pulmonary vascular properties}

Acetylcholine applied locally to the pulmonary arteries has been used for measuring pulmonary flow reserve using a flow wire catheter $(6,18)$. Acetylcholine induces endothelial NO release, leading to relaxation of muscularized small pulmonary vessels. Most recently MRI as well as intravascular ultrasound and tissue doppler assessment of pulmonary distensibility have been shown to potentially ad additional information to pulmonary vascular resistance and vasoreactivity and may predict outcome (18-21).

However, if these newer techniques have the potential to replace conventional invasive measurements and vasoreactivity testing remains unclear.

\section{Pitfalls of catheterization}

PAWP is helpful in differentiating precapillary and postcapillary $\mathrm{PH}$, in particular left heart disease as a potential cause for the $\mathrm{PH}$. A correct position of the catheter tip for a reliable pressure curve with a- and v-wave and in order to avoid over-wedging or under-wedging is crucial to generate reliable measures. Details were discussed previously elsewhere (4).

As discussed above, cardiac output is usually calculated using the Fick's formula with oxygen as tracer substance. Only few centers have the facility to simultaneously measure the oxygen consumption. Equipment needed for exact measurement of oxygen uptake is complex and the procedure itself is time consuming. Therefore, it is important to be aware of the inaccuracy for the calculation of cardiac output by using an assumed oxygen consumption from tables or diagrams.

Although general anesthesia is often used in the pediatric catheterization laboratory, the potential side effects of intubation, mechanical ventilation and anesthetic medication can affect hemodynamic parameters in a way making any reliable assessment impossible. While a $10 \%$ change in PAP can be assumed with the patient spontaneously 
breathing, the odds of PAP caused by general anesthesia may be as high as $20 \%$ without having given any of the AVT drugs. In addition, induction, withdrawal and weaning from general anesthesia are potentially life-threatening stages in this procedure. Particularly in patients with very fragile haemodynamics, only little changes of pulmonary and systemic vascular resistances can have a great impact on haemodynamics and may compromise cardiac output. Recommendations for cardiac catheterization in light to moderate sedation, including a protocol for medication and precautions for troubleshooting, have been published elsewhere (6).

\section{Complication risks}

The risk of complications after cardiac catheterization for $\mathrm{PH}$ has been reported up to $7 \%$ within 24 hours following the procedure (22). An update to the data from this publication found an overall risk of $5.9 \%$, including 5 deaths. Factors with a higher risk for complications were general anesthesia and worse functional class (23). In contrast, adult patients seem to have a lower risk for complications irrespective of general anesthesia or sedation $(24,25)$. A more recent analysis of data from the IMPACT (Improving Adult and Congenital Treatment) registry showed a $1.4 \%$ procedural related risk of death, cardiac arrest or mechanical circulatory support (MCS) and a 5.2\% risk of death before discharge. Younger age, inotropic support and lower systemic arterial saturation were found to be major risk factors. Higher numbers of invasive procedures in $\mathrm{PH}$ patients was found to be associated with a lower risk of death, cardiac arrest or need for MCS (26). The analysis of 6,339 cardiac catheterization procedures in 4,401 individuals from the pediatric health information system database showed a $3.5 \%$ risk of death or MCS with younger age, cardiac surgery during the same hospital stay as the cardiac catheterization, pre-procedural systemic vasodilator treatment and hemodialysis as independent risk factors (27). Consequently, we always have to balance potential risks against the gain of additional information derived from catheterization in particular in pediatric patients. Thus, this invasive procedure should only be performed if the results do have an impact on the clinical decision making process. Invasive assessment should only take place by experienced investigators in experienced centers with appropriate post-procedure monitoring and intensive care backup, ideally with the possibility to apply MCS.

\section{Definition of response to AVT}

It is important to emphasize, that positive AVT has major impact on the subsequent decision about medical treatment and specific PAH medication. Cut-off parameters have been derived from studies in patients with IPAH/HPAH. Thus, it is recommended to perform AVT in these patients for decision making in regard to calcium channel blockers for long-term treatment $(6,28,29)$ and as a predictor for outcome and mortality (19).

Despite these fundamental consequences, a generally accepted definition which parameters classify a patient to a 'responder' with an AVT positive response remains subject to discussions (6). In contrast to the widespread believe that a "positive" AVT is much more likely in children, a recent study has shown no significant differences for adult and pediatric patients, when similar criteria for AVT were used in both age groups (30). Using these parameters for AVT (31) a positive response is defined by a decrease in mPAP by at least $10 \mathrm{mmHg}$ to a value of $<40 \mathrm{mmHg}$ with sustained cardiac output. In patients with $\mathrm{mPAP}<40 \mathrm{mmHg}$ at baseline, positive response is defined by a drop of at least $10 \mathrm{mmHg}$ without a decrease in cardiac output. The German Pediatric Pulmonary Vascular Disease Network suggested a modification of the Barst criteria for AVT (6). These criteria are based on the criteria published by Barst in 1986 (32). The modified Barst criteria are defined for patients with IPAH/HPAH as a $20 \%$ decrease in mPAP and indexed PVR and indexed SVR ratio without a decrease in cardiac output (6). Alternatively, the same authors suggest using the diastolic pressure gradient or a ratio of diastolic PAP/SAP $<0.4$.

It has been shown that about half of the positively tested subjects according to the Barst criteria show longterm clinical and hemodynamic improvement (32). It is important to keep these patients under close follow-up with repeat cardiac catheterizations including an AVT, as these responders may lose their pulmonary vascular responsiveness over time and a change and adjustment of therapy may be required in individual patients.

Other factors like clinical deterioration of patients' functional status and worsening of other clinical and echocardiographic parameter have to be closely observed.

As discussed above, no clear definition exists in the pediatric population and data showing a relationship between longitudinal hemodynamic measures and outcome parameters are lacking (2).

In the group of patients with congenital heart defect 
(APAH-CHD) and associated shunt leading to Qp:Qs >1.5, AVT will be performed with the goal to differ reversible $\mathrm{PAH}$ from irreversible progressive $\mathrm{PAH}$ and for estimation of operability. Despite the fact, that some additional considerations have to be taken into account, no data exists that clearly define hemodynamic parameters to predict operability, and potential reversal of $\mathrm{PAH}$ after surgical closure of shunt lesions (2). Moreover, one has to take into account, that patients with left-to right shunt lesions may not show a decrease in PAP following vasodilator testing and that this condition does not necessarily mean no decrease in PVR long-term. Patients may respond to vasodilator therapy with a decrease in PVR and subsequent increase in pulmonary blood flow but without an effect on PAP, like discussed above.

It has been suggested to define a positive AVT response as a $>20 \%$ fall in indexed pulmonary vascular resistance and a PVRi to values $<6$ indexed Wood units and a PVRi/SVRi ratio of $<0.3$, respectively $(33,34)$. In addition, the diastolic TPG and $\mathrm{dPAP} / \mathrm{dSAP}$ ratio are values that should be looked at.

However, reversal of PAH cannot be reliably predicted, if a specific patient is tested and does not fulfil the above criteria for likelihood of 'operability'. There is a 'grey zone' in the range of a PVRi between 6 and 8 Wood units and PVRi/SVRi 0.3 to 0.5 (34), where successful correction without long-term progressive pulmonary vascular disease may be achievable. However, no clear data exists on the benefit of the shunt correction on long-term outcome after successful repair. In case $\mathrm{PH}$ persists or progresses prognosis may be worse than for a patient with an anatomical shunt in a process turning into an Eisenmenger physiology. Furthermore, the amount of blood flow to the pulmonary and systemic circulation and any change in shunt volume may be influencing the response to targeted PAH treatment. Factors like age, type of cardiac defect, oxygen saturations at rest and under physical exercise, clinical history and other comorbidities, have been shown to influence the outcome following surgery (2).

\section{Diastolic pressure}

Significance of TPG calculated as (pulmonary artery pressure) - (left atrial pressure/capillary wedge pressure) in contrast to the diastolic transpulmonary pressure gradient calculated as (pulmonary artery diastolic pressure) (left atrial pressure) has been discussed previously, since the latter reflects pulmonary vascular resistance in the absence of pulmonary valve regurgitation. The dTPG offers a probably more reliable estimation of the degree of pulmonary vascular disease and pulmonary vascular resistance as it is less affected by vessel elasticity or stiffness and independent of circulatory parameters like right ventricular stroke volume, pulmonary artery flow, cardiac output and left ventricular enddiastolic pressure $(35,36)$.

A diastolic pulmonary pressure gradient (dTPG) equal or less than $3 \mathrm{mmHg}$ is assumed to be normal. In particular in patients with PAH due to left heart disease (LHD$\mathrm{PAH})$ the diastolic transpulmonary pressure gradient can be helpful in detecting any additional precapillary component contributing to PAH. Inconstant conclusions have been made using values for dTPG (= DPD) above $7 \mathrm{mmHg}$ in the adult population. Some authors have found a relationship with a worse outcome (37), however this could not be confirmed in a more recent study (38).

In the updated consensus statement on the diagnosis and treatment of pediatric PH, DPD has been added as an adjunct criterion for detecting pre-capillary and postcapillary $\mathrm{PH}$ or any precapillary component contributing to $\mathrm{PH}$ in left heart disease (3).

Although obstruction of left heart inflow and left heart outflow is a common finding in complex congenital cardiac defects, little is known about left ventricular myocardial dysfunction and the contribution to $\mathrm{PH}$ in children. In addition, impaired left ventricular myocardial performance can also be found in premature babies with related pulmonary problems like bronchopulmonary dysplasia and PPHN. Unfortunately, it remains unclear if the adult criteria can be transferred to the pediatric population since comparable data are lacking.

\section{Single ventricle circulation}

The assessment of pulmonary hypertensive vascular disease in a functional single ventricle circulation needs to be specifically discussed, as these patients do not fit into the regular definition for $\mathrm{PH}$ with a $\mathrm{mPAP}>20 \mathrm{mmHg}$.

The integrity of an intact Fontan physiology is dependent on a low TPG and low pulmonary vascular resistance. Even a small rise in pulmonary vascular resistance can detrimentally impact on the Fontan physiology. These palliated patients with univentricular hearts may develop pulmonary hypertensive vascular disease at all ages and different surgical stages. Even after Fontan completion, pulmonary hypertensive vascular disease may develop later in life. The exact pathophysiological mechanisms are not completely understood but do have a major impact on 
patients' morbidity and mortality. It is believed that nonpulsatile pulmonary blood flow is leading to a very specific entity of pulmonary vascular disease not comparable to any of the other forms (39). Since it is very difficult to find a clear definition of $\mathrm{PH}$ in this special condition these patients were summarized in the group of "segmental $\mathrm{PH}$ " in the 2013 classification of the WSPH. Following the $6^{\text {th }} \mathrm{WSPH}$ these patients were included to the Group 5 classification together with hematologic, metabolic and systemic disorders. The current definition for $\mathrm{PH}$ in single ventricle circulation is a mean TPG $>6 \mathrm{mmHg}$ or PVR index $>3 \mathrm{WU} \cdot \mathrm{m}^{2}$ (Table 1).

\section{Repeat catheterization}

Recommendations and indications for repeat invasive hemodynamic assessment are not well established in pediatric $\mathrm{PH}$. Centers caring for pediatric $\mathrm{PH}$-patients may follow very different policies, depending on experience and protocols.

Indications for follow-up catheterization may vary from assessment of treatment effect or failure of treatment with aggravation of clinical symptoms and disease progression. Despite the lack of data, judgement of hemodynamics is also used for prediction of prognosis and often required as part of the listing process for lung transplantation. However, to date, no association has been shown between changes in hemodynamic parameters and clinical outcome (2). Current recommendations for timing of repeat right heart catheterization vary from every 6 to 12 months in the adult population $(40,41)$ and every 12 to 24 months in pediatric patients (3). The most important question prior to any cardiac catheterization has to be, how the gained results influence the clinicians' counselling and decision making process and potentially alter the patients' clinical management.

\section{Conclusions}

RHC remains the invasive gold standard for confirming the diagnosis of PH. Information gained from RHC is important for identification of possible causes of $\mathrm{PH}$ and mandatory for differentiating between pre- and postcapillary or other mixed forms of the disease. Cardiac catheterization is usually combined with AVT, which is crucial for the decision-making-process for targeted medical therapy. Ideally the test should be performed prior to start of $\mathrm{PH}$ treatment. Despite some fundamental risks, repeat RHC should only be performed, when the acquired hemodynamic parameters will actually impact on medical changes and guide the patients' medical treatment.

\section{Acknowledgments}

Funding: None.

\section{Footnote}

Provenance and Peer Review: This article was commissioned by the editorial office, Cardiovascular Diagnosis and Therapy for the series "Pediatric Pulmonary Hypertension". The article has undergone external peer review.

Conflicts of Interest: The authors have completed the ICMJE uniform disclosure form (available at http://dx.doi. org/10.21037/cdt-20-395). The series "Pediatric Pulmonary Hypertension" was commissioned by the editorial office without any funding or sponsorship. CA and AEL served as the unpaid Guest Editors of the series. CA reports lecture fees from Actelion, outside the submitted work. AEL reports personal fees from Actelion, outside the submitted work. The author has no other conflicts of interest to declare.

Ethical Statement: The authors are accountable for all aspects of the work in ensuring that questions related to the accuracy or integrity of any part of the work are appropriately investigated and resolved.

Open Access Statement: This is an Open Access article distributed in accordance with the Creative Commons Attribution-NonCommercial-NoDerivs 4.0 International License (CC BY-NC-ND 4.0), which permits the noncommercial replication and distribution of the article with the strict proviso that no changes or edits are made and the original work is properly cited (including links to both the formal publication through the relevant DOI and the license). See: https://creativecommons.org/licenses/by-nc-nd/4.0/.

\section{References}

1. Berger RMF, Beghetti M, Humpl T, et al. Clinical features of paediatric pulmonary hypertension: a registry study. Lancet 2012;379:537-46.

2. Rosenzweig EB, Abman SH, Adatia I, et al. Paediatric pulmonary arterial hypertension: updates on definition, 
classification, diagnostics and management. Eur Respir J 2019;53:1801916.

3. Hansmann G, Koestenberger M, Alastalo TP, et al. 2019 updated consensus statement on the diagnosis and treatment of pediatric pulmonary hypertension: The European Pediatric Pulmonary Vascular Disease Network (EPPVDN), endorsed by AEPC, ESPR and ISHLT. J Heart Lung Transplant 2019;38:879-901.

4. Rosenkranz S, Preston IR. Right heart catheterisation: best practice and pitfalls in pulmonary hypertension. Eur Respir Rev 2015;24:642.

5. Douwes JM, van Loon RLE, Hoendermis ES, et al. Acute pulmonary vasodilator response in paediatric and adult pulmonary arterial hypertension: occurrence and prognostic value when comparing three response criteria. Eur Heart J 2011;32:3137-46.

6. Apitz C, Hansmann G, Schranz D. Hemodynamic assessment and acute pulmonary vasoreactivity testing in the evaluation of children with pulmonary vascular disease. Expert consensus statement on the diagnosis and treatment of paediatric pulmonary hypertension. The European Paediatric Pulmonary Vascular Disease Network, endorsed by ISHLT and DGPK. Heart 2016;102:ii23-9.

7. Drossner DM, Kim DW, Maher KO, et al. Pulmonary Vein Stenosis: Prematurity and Associated Conditions. Pediatrics 2008;122:e656-61.

8. del Cerro MJ, Sabaté Rotés A, Cartón A, et al. Pulmonary hypertension in bronchopulmonary dysplasia: Clinical findings, cardiovascular anomalies and outcomes. Pediatr Pulmonol 2014;49:49-59.

9. Kovacs G, Avian A, Pienn M, et al. Reading pulmonary vascular pressure tracings: How to handle the problems of zero leveling and respiratory swings. Am J Respir Crit Care Med 2014;190:252-7.

10. Mullins CE. Cardiac Catheterization in Congenital Heart Disease. Blackwell Publishing Inc; 2006.

11. Hoeper MM, Bogaard HJ, Condliffe R, et al. Definitions and Diagnosis of Pulmonary Hypertension. J Am Coll Cardiol 2013;62:D42-50.

12. Fick A. Über die Messung des Blutquantums in den Herzventrikeln. SB phys-med Ges 1870: Würzburg 16.

13. Gutgesell HP, Williams RL. Caval Samples as indicators of mixed venous oxygen saturation: Implications in atrial septal defect. Cardiovasc Dis 1974;1:160-4.

14. Fakler U, Pauli C, Hennig M, et al. Assumed oxygen consumption frequently results in large errors in the determination of cardiac output. J Thorac Cardiovasc Surg 2005;130:272-6.
15. Li J, Bush A, Schulze-Neick I, et al. Measured versus estimated oxygen consumption in ventilated patients with congenital heart disease: the validity of predictive equations. Crit Care Med 2003;31:1235-40.

16. Rutledge J, Bush A, Shekerdemian L, et al. Validity of the LaFarge equation for estimation of oxygen consumption in ventilated children with congenital heart disease younger than 3 years-A revisit. Am Heart J 2010;160:109-14.

17. Wolf A, Pollman MJ, Trindade PT, et al. Use of assumed versus measured oxygen consumption for the determination of cardiac output using the Fick principle. Cathet Cardiovasc Diagn 1998;43:372-80.

18. Apitz C, Zimmermann R, Kreuder J, et al. Assessment of pulmonary endothelial function during invasive testing in children and adolescents with idiopathic pulmonary arterial hypertension. J Am Coll Cardiol 2012;60:157-64.

19. Dyer K, Lanning C, Das B, et al. Noninvasive Doppler Tissue Measurement of Pulmonary Artery Compliance in Children with Pulmonary Hypertension. J Am Soc Echocardiogr 2006;19:403-12.

20. Ploegstra MJ, Zijlstra WMH, Douwes JM, et al. Prognostic factors in pediatric pulmonary arterial hypertension: A systematic review and meta-analysis. Int J Cardiol 2015;184:198-207.

21. Berger RMF, Cromme-Dijkhuis AH, Hop WCJ, et al. Pulmonary Arterial Wall Distensibility Assessed by Intravascular Ultrasound in Children With Congenital Heart Disease: An Indicator for Pulmonary Vascular Disease? Chest 2002;122:549-57.

22. Gan CTJ, Lankhaar JW, Westerhof N, et al. Noninvasively Assessed Pulmonary Artery Stiffness Predicts Mortality in Pulmonary Arterial Hypertension. Chest 2007;132:1906-12.

23. Beghetti M, Berger RMF, Schulze-Neick I, et al. Diagnostic evaluation of paediatric pulmonary hypertension in current clinical practice. Eur Respir J 2013;42:689-700.

24. Beghetti M, Schulze-Neick I, Berger RMF, et al. Haemodynamic characterisation and heart catheterisation complications in children with pulmonary hypertension: Insights from the Global TOPP Registry (tracking outcomes and practice in paediatric pulmonary hypertension). Int J Cardiol 2016;203:325-30.

25. D'Alto M, Dimopoulos K, Coghlan JG, et al. Right Heart Catheterization for the Diagnosis of Pulmonary Hypertension: Controversies and Practical Issues. Heart Fail Clin 2018;14:467-77.

26. Hoeper MM, Lee SH, Voswinckel R, et al. Complications 
of Right Heart Catheterization Procedures in Patients With Pulmonary Hypertension in Experienced Centers. J Am Coll Cardiol 2006;48:2546-52.

27. O'Byrne ML, Kennedy KF, Kanter JP, et al. Risk Factors for Major Early Adverse Events Related to Cardiac Catheterization in Children and Young Adults With Pulmonary Hypertension: An Analysis of Data From the IMPACT (Improving Adult and Congenital Treatment) Registry. J Am Heart Assoc 2018;7:008142.

28. O'Byrne ML, Glatz AC, Hanna BD, et al. Predictors of Catastrophic Adverse Outcomes in Children with Pulmonary Hypertension Undergoing Cardiac Catheterization: A Multi-Institutional Analysis From the Pediatric Health Information Systems Database. J Am Coll Cardiol 2015;66:1261-9.

29. Hansmann G, Apitz C. Treatment of children with pulmonary hypertension. Expert consensus statement on the diagnosis and treatment of paediatric pulmonary hypertension. The European Paediatric Pulmonary Vascular Disease Network, endorsed by ISHLT and DGPK. Heart 2016;102:ii67-85.

30. Douwes JM, Humpl T, Bonnet D, et al. Acute Vasodilator Response in Pediatric Pulmonary Arterial Hypertension Current Clinical Practice From the TOPP Registry. J Am Coll Cardiol 2016;67:1312-23.

31. Sitbon O, Humbert M, Jaïs X, et al. Long-term response to calcium channel blockers in idiopathic pulmonary arterial hypertension. Circulation 2005;111:3105-11.

32. Barst RJ. Pharmacologically induced Pulmonary Vasodilatation in Children and Young Adults with Primary Pulmonary Hypertension. Chest 1986;89:497-503.

33. Yung D, Widlitz AC, Rosenzweig EB, et al. Outcomes in

Cite this article as: Kaestner M, Apitz C, Lammers AE. Cardiac catheterization in pediatric pulmonary hypertension: a systematic and practical approach. Cardiovasc Diagn Ther 2021;11(4):1102-1110. doi: 10.21037/cdt-20-395 children with idiopathic pulmonary arterial hypertension. Circulation 2004;110:660-5.

34. Ivy DD, Abman SH, Barst RJ, et al. Pediatric Pulmonary Hypertension. J Am Coll Cardiol 2013;62:D117-26.

35. Kozlik-Feldmann R, Hansmann G, Bonnet D, et al. Pulmonary hypertension in children with congenital heart disease (PAH-CHD, PPHVD-CHD). Expert consensus statement on the diagnosis and treatment of paediatric pulmonary hypertension. The European Paediatric Pulmonary Vascular Disease Network, endorsed by ISHLT and DGPK. Heart 2016;102:ii42-8.

36. Naeije R, Vachiery JL, Yerly P, et al. The transpulmonary pressure gradient for the diagnosis of pulmonary vascular disease. Eur Respir J 2013;41:217-23.

37. Vachiéry JL, Adir Y, Barberà JA, et al. Pulmonary Hypertension Due to Left Heart Diseases. J Am Coll Cardiol 2013;62:D100-8.

38. Gerges C, Gerges M, Lang IM. Characterization of pulmonary hypertension in heart failure using the diastolic pressure gradient. The conundrum of high and low diastolic pulmonary gradient. JACC Heart Fail 2015;3:424-5.

39. Tampakakis E, Leary PJ, Selby VN, et al. The Diastolic Pulmonary Gradient Does Not Predict Survival in Patients With Pulmonary Hypertension Due to Left Heart Disease. JACC Heart Fail 2015;3:9-16.

40. Ridderbos FJS, Wolff D, Timmer A, et al. Adverse pulmonary vascular remodeling in the Fontan circulation. J Heart Lung Transplant 2015;34:404-13.

41. Galiè N, Humbert M, Vachiery JL, et al. 2015 ESC/ERS Guidelines for the diagnosis and treatment of pulmonary hypertension. Eur Heart J 2016;37:67-119. 